IV. Aus dem städtischen Krankenhause Friedrichshain, Abtheilung des Herrn Prof. Dr. Fürbringer in Berlin.

\section{Zur klinischen Würdigung des Chloralamids und des Somnals.}

Von Dr. Adolf Robinson, Assistenzarzt.

Die praktische Medicin vermag in der Lösung der wichtigen und alltäglichen Aufgabe der Behandlung der Schlaflosigkeit bei aller Anerkennung des hohen Werthes der Erfüllung der Indicatio causalis durch nicht-medicamentöse Maassnahmen der eigentlichen Arzneimittel nicht zu entrathen. Diese Erkenntniss hat im Verein mit der durch tausend und abertausend Erfahrungen gefestigten Thatsache, dass keins unserer Schlafmittel, auch nicht das beste, mehr oder weniger unliebsame, sogar bedenkliche Nebenwirkungen in den Kreis seiner Erfolge zu führen, zu vermeiden imstande war, eine wahre Jagd nach neuen Arzneikörpern zur Bekämpfung der Insomnie eröffnet, deren Ausbeute unseren Arzneischatz in werthvoller Weise bereichert hat, aber als vollkommen nicht gelten darf. Einen correcten Begriff und übersichtliche Zusammenstellung dessen, was heutzutage die Fortschritte der Medicin gezeitigt, giebt uns Jastrowitz in seinem neuesten Aufsatz über die Behandlung der Schlaflosigkeit (Deutsche med. Wochenschr. 1889, No. 31-34).

Aus den Ausführungen dieses erfahrenen Autors erhellt, dass weder die Opiate noch das Chloral - diese beiden Mittel stehen auch noch heutzutage obenan - , weder das Paraldehyd noch das Amylenhydrat, weder das Sulfonal noch das Urethan den Haupterfordernissen: Promptheit der Wirkung und Unschädlichkeit anstandslos genügen. Unter solchen Umständen ist die Einführung neuer rationeller Mittel zur Behandlung der Agrypnie nur gerechtfertigt und $\mathrm{zu}$ begrüssen.

Gern habe ich mich daher der mir von Herrn Prof. Fï r bringer gestellten Aufgabe, mit dem Chloralamid und dem Somnal an Patienten seiner Wahl der inneren Station unseres Krankenhauses vergleichende Prüfungen über die Wirkungsweise bei den verschiedensten Leiden vorzunehmen und über die Resultate zu berichten. unterzogen.

Meinem hochverehrten Chef erstatte ich an dieser Stelle meinen verbindlichsten Dank für die Unterstïtzung und Controlle bei dieser Arbeit.

Das aus der chemischen Fabrik von E. Schering stammende Chloralamid hat inzwischen eine kleine Litteratur veranlasst, während über die Wirkungen des vom Apotheker S. Radlauer dargestellten Somnals (äthylirten Chloral-Urethans) meines Wissens noch keine speciellen Publicationen erfolgt sind.

Das einzige, was ich gefunden habe, besteht in einem dem Prospecte beigegebenen Zeugniss von P. Guttmann, dass bei den von ihm angestellten Untersuchungen schlafbringende Wirkung ,in den meisten Fällen" beobachtet worden sei, sowie in einer Anzeige der Kronenapotheke, dass ausserdem das Somnal in der Königlichen Universitätspoliklinik und Charité, von $\mathrm{Krafft-Ebing}$ und $\mathrm{Za}$ gorski "mit gutem Erfolg" angewendet worden sei.

Das Chloralamid besteht aus weissen, kleinen Crystallen; das Somnal ist eine wässerige, farblose, spirituös riechende Flüssigkeit. Der Preis beider Mittel pro Schlafdose beträgt etwa 10 Pfennig.

Ein Theil des Somnals wurde uns von Herrn Radlauer freundlichst zur Verfügung gestellt. Der Geschmack des Chloralamids ist im ganzen nicht unangenehm, gering bitter, der des Somnals kratzend und bitterer; doch muss man selbst von letzterem Mittel zugeben, dass es angenehmer, wie das Chloralhydrat einzunehmen ist.

Aus den die Bedeutung des Chloralamids als Schlafmittels aus eigener Erfahrung besprechenden, grösstentheils erst während unserer Versuche veröffentlichten Arbeiten erwähne ich - um nur das klinisch Wichtige zu berühren - dass fast alle Autoren ${ }^{1}$ ) sich

1) 1) Jastrowitz, Ueber die Behandlung der Schlaflosigkeit. Deut. med. Wochenschr. 1889, No. 31-34. 2) Hagen und Ḧ̈fler, Ueber die schlafmachende: Wirkung des Chloramids. Münch. med. W Wochenschr. 1889, No. 30. 3) Lettow, Ueber Chloralamid als Hypnoticum, Inauguraldissertion gemeinsam dahin aussprechen, dass Chloralamid kein Narcoticum, sondern nur ein Hypnoticum ist, dass Nebenwirkungen auf die Herzthätigkeit, auf den Respirationsapparat fast ganz fehlen - Hagen und Hüfler erwähnen einen Collapszustand, den sie aber auch nur beschränkt dem Mittel zuschreiben - auf den Digestionsapparat geringe, bisweilen häufigere, bisweilen seltenere Wirkungen in die Erscheinung treten.

Jastrowitz, welcher „keine psychische Erregung, wie nicht selten beim Chloral", gesehen, giebt Erbrechen und häufigeren Kopfschmerz als Folge der Medication an.

Die Verabreichung der Mittel geschah beiderseits in Lösung, und zwar derart, dass je ein Esslöffel $1 \mathrm{~g}$ des Mittels enthielt. Als Geschmackscorrigens wurde Succ. Liquir. hinzugesetzt.

Die Patienten erhielten meist den Schlaftrunk Abends $8 \mathrm{Uhr}$; einige, die ich während der Nachtvisite wachend fand, auch mitten in der Nacht.

Ess wurde stets nach den üblichen Grundsätzen bei Prüfung von Schlafmitteln verfahren. Vor allem wussten die Patienten nichts davon, dass die ihnen verabreichte Medicin Schlaf bewirken sollte.

Bei einer grösseren Anzahl von Kranken wurde vor der Eingabe die Temperatur gemessen, der Puls und die Respiration gezählt, darauf nach ein und zwei Stunden eine Wiederholung del Bestimmung vorgenommen. Auch das specifische Gewicht des Urins, sowie die Menge wurde in mehrereren Fällen genau bestimmt.

Fünfzig verschiedene Patienten erhielten jedes Mittel, und damit die Prüfung der Wirkung sich zuverlässiger gestalte, wurden einer grösseren Anzahl derselben einen Tag Somnal, einige Tage darauf Chloralamid und dann zur Vergleichung Chloralhydrat oder Morph. mur. verabreicht. Nur Kranke, von denen man überzeugt sein konnte, dass sie ohne Anwendung eines Medicamentes nicht einschlafen würden, erhielten dasselbe. Um zu erfahren, ob beide Mittel als Narcotica oder nur als Hypnotica wirken, d. h. ob dieselben etwa wie das Morphium unter allen Umständen den Schlaf erzwingen, oder nur ein vorhandenes Schlafbedürfniss befriedigen helfen, wurden vier Patienten wiederholt mit beiden Medicamenten behandelt.

Ich will zunächst, ohne mein Resultat vorweg zu nehmen, den Abdruck einiger meiner Versuchsprotokolle vorlegen, damit der Leser aus denselben über die Berechtigung der gezogenen Schlüsse sich ein eigenes Urtheil bilden kann.

1. Gruppe: Agrypnie wegen körperlicher Beschwerden, Schmerzen, Husten etc.

I. Ka iser, 55jähriger Mann Phthisis pulm. Schmerzen und Husten.

10. September. Abends $8 \mathrm{Uhr} 2 \mathrm{~g}$ Somnal. Schläft erst um $11 \mathrm{Uhr}$ ein, wacht um 12 Uhr auf und wacht dann die ganze Nacht.

11. September. Fühlt sich unverändert, hat keine Beschwerden.

12. September. Abends 8 Uhr $2 \mathrm{~g}$ Chloralamid; schläft $8^{1 / 2} \mathrm{Uhr}$ eill, wacht um $10 \mathrm{Uhr}$ auf, ist sehr aufgeregt, will fortwährend aus dem Bett. Puls sehr beschleunigt, klein.

13. September. Klagt über starke Kopfschmerzen und Benommenheit.

14. September. Abends $8 \mathrm{Uhr} 2 \mathrm{~g}$ Chloralhydrat; schläft $8^{1 / 2} \mathrm{Uhr}$ ein, schläft bis 2 Uhr, dann von 3 Uhr bis 10 Uhr Vormittags sehr fest. Puls beschleunigt, klein.

II. Friedemann, 55jähriger Mann, Phthisis pulm. und Epilepsia. Husten.

6. September. Abends 8 Uhr $2 \mathrm{~g}$ Somnal, scbläft 9 Uhr 30 Min. ein, wacht um $11 \mathrm{Uhr}$ auf durch Erbrechen, kann nicht wieder einschlafen. 8 Uhr Abends 37.8, Puls 96, Resp. 29, Urin spec. Gewicht 1012. 9 Uhr Abends Puls 96, Temp. 38,1, 10 Uhr Abends Puls 88, Temp. 38,2.

7. September. Pat. fühlt sich sehr erquickt, hat keine besonderen Beschwerden. Urin Morgens spec. Gewicht 1017.

11. September. Abends $8 \mathrm{Uhr} 1 \mathrm{~g}$ Chloralamid; schläft nicht, $9 \mathrm{Uhr}$ wieder $1 \mathrm{~g}$ Chloralamid, schläft von $9^{1 / 2} \mathrm{Uhr}$ bis $12 \mathrm{Uhr}$, bekommt dann das dritte Gramm Chloralamid und erwacht nicht bis Morgens 5 Uhr. 8 Uhr Abends Temp. 37,6, Puls 84, Resp. 28. 9 Uhr Abends Temp. 37,8, Puls 78, Resp. 28. 10 Uhr Abends desgleichen.

12. September. Bricht mehrere Male, fühlt sich sehr unwohl und benommen, während er sonst noch nie gebrochen.

13. September. Abends $8 \mathrm{Uhr} 2 \mathrm{~g}$ Somnal; schläft fast die ganze Nacht ohne Unterbrechung.

17. September. Abends $8 \mathrm{Uhr} 2 \mathrm{~g}$ Chloralamid; schläft ein um $9^{1 / 2}$ Uhr, 8 Uhr Puls 92, Resp. 24, Temp. 38,0. 9 Uhr Puls 94, Resp. 24, Temp. 37,8. Schlaf unruhig, lejse, wacht bei der geringsten Störung auf.

18. September. Vormittag starke Kopfschmerzen. Schläft Abends gar nicht, bekommt um 12 Uhr Nachts $2 \mathrm{~g}$ Chloralamid, schläft nach einer halben Stunde ein, wacht um 2 Uhr auf und bleibt wach. Patient schläft jetzt nach 0,01 Morph. subcutan stets ohne Unterbrechung die ganze Nacht.

Greifswald 1889. 4) Reichmann, Ueber Chloralamid, ein neues Schlafmittel. Deut. med. Wochenschr. 1889, No. 31. 5) Pe iper, Chloralamid, ein neues Schlafmittel. Deut. med. Wochenschr. 1889, No. 32. 6) Rabow, Ueber Chloralamid, ein neues Hypnoticum. Centralblatt für Nervenheilkunde 1889, No. 15. 7) Hagemann und Strauss, Ueber Chloralamid. Berl. klin. Wochenschr. 1889, No. 33. 8) Alt, Chloralamid, ein neues Schlafmittel. Berl. klin. Wochenschr. 1889, No. 36. 9) $\mathrm{Kny}$, Chloralamid, ein neues Schlafmittel. Therápeut. Monatshefte, August: 1889. 
III. Schnell, 43 jähriger Mann Phthisis pulm. Husten.

9. September. Abends $6 \mathrm{Uhr} 2 \mathrm{~g}$ Somnal. $8^{1 / 2} \mathrm{Uhr}$ eingeschlafen wacht fortwährend auf, ist beim kleinsten Geränsch wach, bekommt um 12 Uhr wieder $1 \mathrm{~g}$, schläft dann gar nicht mehr. 8 Uhr Abends Temp. 38,7, Puls 96, Resp. 22. 9 Uhr Abends Temp. 38,4, Puls 94, Resp. 22. 10 Uhr Abends Temp. 38,3, Puls 90, Resp. 22.

10. September. Vormittags klagt über Mïdigkeit.

12. September. Abends 8 Uhr $2 \mathrm{~g}$ Chloralamid. Scliläft um $9^{1 / 4}$ Uhr ein, erwacht um $10^{3} / 4 \mathrm{Uhr}$, delirirt die ganze Nacht, spricht fortwährend ungereimtes $Z$ eug. 8 Uhr Abends Temp. 39,9, Puls 88, Resp. 22. 9 Uhr Abends Temp. 38,1, Puls 100, Resp. 22. 10 Uhr Abends Temp. 37,8, Puls 102, Resp. 22.

13. Septemher. Vormittags delirirt fort, antwortet ganz verkehrt auf Fragen.

14. September. Pat. weigert sich entschieden, eins der beiden Schlafmittel zu nehmen, bittet dagegen um Chloralhydrat mit Morph. mur., nach welchem er stets die Nacht ohne Störung durchschläft.

IV. Bartig, 19jähriger Mann. Ulcus ventric. Polyarthritis rheumat. Schmerzen.

9. September. Abends 8 Uhr 2 g Somnal per Rectum. Schläft ein um $81 / 2$ Uhr, schläft die Nacht gut durch. 8 Uhr T. 38,8, P. 84, R. 20. 9 Uhr T. 38,6, P. 82 , R. 22 . 10 Uhr 'T. 38,5 , P. 78 , R. 20.

10. September. Klagt über Kopfschmerzen, fühlt sich „duselig“"

14. September. Abends 8 Uhr $2 \mathrm{~g}$ Chloralamid per Rectum, schläft $1108^{3} / 4$ Uhr ein, wacht um $12^{1 / 2}$ Uhr auf und ist von da ab sehr unruhig, der Puls ist so klein, dass 2 mal Kampher gegeben werden musste. 8 Uhr T. 37,9, P. 100, R. 32 . 9 Uhr T. 37,5, P. 116, R. 34 . 10 Uhr T. 37,5 , P. 132 , R. 40 .

15. September. Pat. hat grosse Beschwerden, fühlt sich schlaff und müde und weiss nicht, was mit ihm passiert.

18. September. 8 Uhr Abends $2 \mathrm{~g}$ Chloralamid, schläft um 8 Uhr 50 ein, wacht um 12 Uhr auf, bricht stark und schläft dann mit Unterbrechungen bis Morgens. In der Zwischenzeit erhielt Pat. Abends Morph. mur. subcutan 0,006 und schläft danach gut.

V. Seegebrecht, 43jähriger Mann. Nephrolithiasis, Pyelonephritis, Myocarditis. Polyarthritis rheum. Schmerzen.

10. September. Abends 8 Uhr $2 \mathrm{~g}$ Somnal. Patient fühlt gar keine Wirkung, schläft die ganze Nacht fast gar nicht.

11. September. Abends 6 Uhr $3 \mathrm{~g}$ Somnal. Pat. bricht 2 mal kurz darauf stark, fühlt grosses Unbehagen, schläft ein und wacht erst Morgens $4 \mathrm{Uhr}$ wieder auf. Schlaf sehr leise.

12. September. Klagt über Uebelkeit und Schwindel, greift stets bei den Gegenständen vorbei.

18. September. Abends 6 Uhr $2 \mathrm{~g}$ Somnal, schläft nicht. Um 9 Uhr wieder $2 \mathrm{~g}$, schläft um $10 \mathrm{Uhr} 30$ ein, wacht erst $4^{1 / 2}$ Uhr auf, abgesehen von kurzen Unterbrechungen.

24. September. Abends 8 Uhr 2 g Chloralamid schläft wesentlich besser wie nach Somnal. 8 Uhr T. 38,3 , P. 64 , R. 16.9 Uhr T. 38,1, P. 64 , R. 20 . 10 Uhr T. 38,0 , P. 64 , R. 20 .

25. September. Klagt über Kopfschmerzen und Müdigkeit. Pat. hat vom 19. bis 24. Morph. mur. (subcut. 0,01) bekommen, wonach er sich bedeutend wohler fühlt wie nach letzterem.

VI. Liepe, 55jähriger Mann. Myocarditis. Schmerzhafte Beklem-

16. September. 8 Uhr Abends 2 g Somnal. Schlief um $8^{1 / 2}$ Uhr ein, ohne wesentliche Unterbrechung. 8 Uhr Temp. 37,1, Puls 60, Resp. 24. 9 Uhr Temp. 37,0, Puls 58, Resp. 24. 10 Uhr Temp. 37,0, Puls 58, Resp. 24.

17. September. Keine Klagen.

18. September. 8 Uhr Abends $2 \mathrm{~g}$ Somnal, schläft gut.

22. September. 8 Uhr Abends 2 G Chloralamid. Schläft 91/2 Uhr ein, wacht fortwährend auf und ist um 4 Uhr ganz wach. Der Puls wird schnell und so klein, dass mehrmals Kampher gegeben werden muss. 8 Uhr Temp. 36,7, Puls 62, Resp. 24. 9 Uhr Temp. 36,9, Puls 78, Resp. 26. 10 Uhr Temp. 36,9, Puls 100, Resp. 29.

23. September. Vorm. Puls wieder 66 und gut. Abends 8 Uhr $1 \mathrm{~g}$ Chloralamid, schläft wenig. Puls wird diesmal nicht alteriert.

VII. Fischer, 39 jähriger Mann. Phthisis pulmon. Schmerzen und Husten.

15. September. Abends 8 Uhr $2 \mathrm{~g}$ Somnal. Schläft erst um $9 \frac{1}{2}$ Uhr ein, ist die Nacht unruhig, wacht bei der geringsten Störung auf

16. September. Patient hat Kopfschmerzen und behauptet, so schlecht noch nie im Krankenhause geschlafen zu haben, wie diese Nacht (vorher bekam er Chloralhydrat mit Morph. mur.).

24. September. Aussprüche des Patienten: „Ich bin ganz verrückt im Kopf. Das Mittel kann ich nicht nocheinmalnehmen, solche Kopfschmerzen habe ich noch nie gehabt."

VIII. Toepel, 54jähriger Mann. Beschwerdevolle Pericarditis exsud. Polyarthritis rheum.

20. September. Abends 8 Uhr $2 \mathrm{~g}$ Somnal. Pat. schläft $8^{1 / 2}$ Uhr ein. Schlaf sehr leise, wacht öfter auf, schläft gleich wieder ein. 8 Uhr Temp. 38,8, Puls 64, Resp. 18. 9 Uhr Temp. 38,1, Puls 62, Resp. 18. 10 Uhr Temp. 37,9 , Puls 56, Resp. 18.

21. September. Fühlt sich erquickt und hat keine besonderen Beschwerden.

24. September. Abends 8 Uhr $1 \mathrm{~g}$ Chloralamid. Patient schläft um $8^{1 / 2}$ Uhr ein. Schlaf sehr leise, wacht um $1^{1 / 2}$ Uhr auf, ist ziemlich stark cyanotisch. Puls kaum fühlbar, während vorher derselbe langsam kräftig und rhythmisch war. Erst nach 4 Spritzen 0l. camphor. lässt die Angst nach, Puls wird kräftiger. 8 Uhr Temp. 37,8, Puls voll, regelmässig, 60, Resp. 24. 9 Uhr Temp. 37,6, Puls 58, Resp. 24. 10 Uhr Temp. 37,6, Puls 58, Resp. 22. $1^{1 / 2}$ Uhr Temp. 37,9, Puls 50, kaum fühl- bar, Resp. 32. 4 Uhr Temp. 37,9, Puls 72 (nach ol. camphor besser), Resp. 28.

25. September. Puls gut. Ausser starken Kopfschmerzen kein Unbehagen. Patient hat bis jetzt jeden Abend 0,006 Morph. mur. subcutan hekommen, ohne dass einmal hedeutendere Einwirkingen auf's Herz bemerkt wurden, und schlief leidlich danach.

IX. We is e, 60jähriger Mann. Carcinoma ventriculi, Schmerzen, Schlaflosigkeit.

14. September. $8 \mathrm{Uhr}$ Abends $2 \mathrm{~g}$ Somnal. Patient schläft gar nicht, klagt über Uebelkeiten, erhält um 10 Uhr ein drittes Gramm; schläft um 10\% Uhr ein, wacht durch Erbrechen (früher noch nie erbrochen) IIm 12 Uhr auf, weiterhin gar kein Schlaf mehr.

15. September. Fãllt beim Aufstehen um, hat grosse Kopfschmerzen.

19. September. $8 \mathrm{Uhr}$ Abends $2 \mathrm{~g}$ Chloralamid. Patient schläft um 9 Uhr ein, wacht um 1 Uhr auf, schläft nicht mehr, sehr unruhig.

20. September. Stärkere Magenschmerzen wie sonst. Patient schläft stets die ganze Nacht hindurch nach 0,06 Morph. mur. subcutan.

$\mathrm{X}$. Wied eberg, 26jähriger Mann. Intoxicatio saturnina. Starke Schmerzen, Schlaflosigkeit.

18. September. $8 \mathrm{Uhr}$ Ahends $2 \mathrm{~g}$ Somnal. Patient schläft gar nicht ein, klagt über so grosse Schmerzen, dass ihm, nachdem er um 9 Uhr noch $1 \mathrm{~g}$ erhalten und anch dieses bis $12 \mathrm{Uhr}$ nicht gewirkt hatte, um $121 / 2 \mathrm{Uhr}$ eine Spritze Morph. mur. 0,01 gegeben werden musste, wonach er um $12^{3} / 4$ einschlief.

20. September. $8 \mathrm{Uhr}$ Abends $2 \mathrm{~g}$ Chloralamid. Patient klagt bald darauf über grössere Schmerzen, bricht innerhalb einer Stunde $4 \mathrm{mal}$, während er sonst den ganzen Tag höchstens 3 mal bricht. Dieselben werden so heftig, dass um $12 \mathrm{Uhr}$ wieder Morph. mur. angewendet werden muss.

2. Gruppe: Schlaflosigkeit infolge von acuten Infectionskrankheiten ohne wesentliche Beschwerden und A ufregung.

XI. Czypkus, 18jähriger Mann. Typhus, abdom. Nicht benommen. Schlaflosigkeit.

12. September. Abends 8 Uhr $2 \mathrm{~g}$ Somnal. Patient schläft $81 / 2 \mathrm{Uhr}$ ein, wacht fortwăhrend auf, Schlaf sehr leise, aber fühlt sich am Morgen etwas erquickt. 8 Uhr Temp. 39,8, Puls 108, Resp. 28. 9 Uhr Temp. 39,8, Puls 104, Resp. 28. 10 Uhr Temp. 38,8, Puls 96, Resp. 24.

13. September. Pat. $2 \mathrm{~g}$ Somnal schläft mit Unterbrechungen die ganze Nacht.

14. September. Pat. $2 \mathrm{~g}$ Somnal, wacht schon um $11 \mathrm{Uhr}$ auf, kann nicht wieder einschlafen, erhält um $\mathbf{l}$ Uhr das dritte Gramm. Schläft gar nicht, grosses Angstgefühl, benommen. Puls 108, aber ziemlich kräftig, um 10 Uhr Puls 100.

15. September. Pat. bricht $2 \mathrm{mal}$ (vorher nie gebrochen), klagt über heftige Magenschmerzen.

17. September. Abends 8 Uhr $1 \mathrm{~g}$ Chloralamid. Pat. schläft nur von $12-2$ Uhr, sonst ist er ganz wach.

18. September. Abends $8 \mathrm{Uhr} 2 \mathrm{~g}$ Chloralamid. Pat. wird sehr aufgeregt, ist kaum im Bette zll halten, delirirt die ganze Nacht. 8 Uhr Temp. 39,2, Puls 98, Resp. 28. 9 Uhr Temp. 39,1, Puls 90, Resp. 28. 10 Uhr Temp. 39,6, Puls 112, Resp. 32.

XII. Andrzejewski, 25 jähriger Mann. Typhus abdom. Nicht benommen.

15. September. Abends 8 Uhr $2 \mathrm{~g}$ Somnal. Pat. schläft mit grösseren Unterbrechungen die ganze Nacht. 8 Uhr Temp. 39,7, Puls 96, Resp. 24. 9 Uhr Temp. 39,6, Puls 96, Resp. 24. 10 Uhr Temp. 39,4, Puls 90, Resp. 24.

16. September. Abends 8 Uhr 2 g Somnal. Pat. schläft wenig, bekommt um $12 \mathrm{Uhr}$ noch ein drittes Gramm. Pat. schläft jetzt gar nicht mehr, ist sehr aufgeregt, Puls beschleunigt 108 (vorher 84 Pulse).

18. September. Abends $8 \mathrm{Uhr} 1 \mathrm{~g}$ Chloralamid. Pat. fïhlt gar keine Wirkung, schläft gar nicht.

19. September. Abends 8 Uhr $1^{1 / 2}$ g Chloralamid. Pat. ist unruhig schläft $a b$ und zu eine halbe Stunde, wirft sich im Bette und spricht öfters ungereimtes Zeug. 8 Uhr Temp. 39,9, Puls 94, Resp. 24. 9 Uhr Temp. 39,9, Puls 91, Resp. 22. 10 Uhr Temp. 39,7, Puls 88, Resp. 22. 2 Uhr Temp. 38,6, Puls 98, Resp. 26.

20. September. Abends 8 Uhr $2 \mathrm{~g}$ Chloralamid. Pat. schläft um 9 Uhr ein, wacht um $11 \mathrm{Uhr}$ auf, ist ganz benommen, Puls um $12 \mathrm{Uhr}$ so klein, dass stündlich bis zum Morgen Campher gegeben werden muss. 8 Uhr Temp. 39,7, Puls 98, Resp. 26. 9 Uhr Temp. 39,1, Puls 94 Resp. 24. 10 Uhr Temp. 39,0, Puls 98, Resp. 24. 12 Uhr Temp. 38,5, Puls 120, Resp. 32.

22. September. 1 g Sulfonal, 6stündiger ununterbrochener Schlaf.

XIII. Held, 23 jähriger Mann. Typhus abdom. Nicht benommen.

10. September. Ahends 8 Uhr $2 \mathrm{~g}$ Somnal. Pat. schläft die Nacht ut mit kleinen Unterbrechungen. Schlaf sehr leise.

11. September. $2 \mathrm{~g}$ Somnal. Pat. schläft etwas unruhig, wacht sehr oft auf.

12. September. $2 \mathrm{~g}$ Somnal. Pat. schläft um $8^{1 / 2}$ Uhr ein. Der Schlaf ist unruhig, leise, nicht erquickend.

13. September. Pat. schläft die Nacht gut, ohne aufzuwachen.

17. September. Abends 8 Uhr 2 g Chloralamid. Pat. fühlt keine Hüdigkeit, schläft höchstens 2 Stunden mit Unterhrechung die ganze Nacht.

18. September. 1/1/2 g Chloralamid. Pat. ist aufgeregt, müde, schläft sehr wenig.

19. September. 2 g Chloralamid. Pat. schläft gegen $9 \mathrm{Uhr}$ ein, wacht um 11 Uhr auf, ruft und schreit fortwährend: "Kinder, was habt ihr mit mir gemacht, mir wird ja schlechter." 8 Uhr Temp. 40,3, Puls 94, Resp. 30. 9 Uhr Temp. 39,8, Puls 90, Resp. 28. 10 Uhr Temp. 39,8, 
Puls 98, Resp. 28. 12 Uhr Temp. 40,0, Puls 108, Resp. 32. Puls um 12 Uhr klein, leise aber nicht bedrohlich.

20. September. Pat. bricht 2 mal und hat starke Kopfschmerzen.

\section{Gruppe: Wirkung bei Aufregungsuständen.}

XIV. Senft, 54jälıiger Mann. Delirium tremens.

22. September. Pat. erhält Abends $3 \mathrm{~g}$ Somnal. Schläft keinen A ugenblick, tobt in der Zelle, wie vorher.

23. September. Pat. erhălt um 8 Uhr $2 \mathrm{~g}$ Somnol, um $10 \mathrm{Uhr}$ wieder $2 \mathrm{~g}$ und 2 Uhr nochmals $2 \mathrm{~g}$. Pat. ist die gauze Nacht unruhig, kein Schlaf. Morgens 6 Uhr schläft Pat. ein bis 9 Uhr. 9 Uhr Temp. 37,s, Puls 84 Resp. 24. 11 Uhr Temp. 37,6, Puls 72, Resp. 18. 1 Uhr Temp. 36,9, Puls 96, Resp. 28.

XV. Berger, 46 jähriger Mann. Delirium tremens.

21. September. Mittags 12 Uhr $2 \mathrm{~g}$ Somnal. Pat. ist sehr unruhig, keine Wirkung zu erkennen, Puls voll und gut 86 . Um 2 Uhr 2 g Somnal. Pat. tobt ruhig fort. Um 6 Uhr Puls 94. Pat. hat noch gar nicht geschlafen; Nachts wird die Unruhe so gross, dass Pat. in die Zelle gebracht werden muss. Gegen Morgen schläft Pat. 3 Stunden.

XVI. Portmann, 52 jähriger Mann. Delirium tremens.

16. September. Abends $8 \mathrm{Uhr} 2 \mathrm{~g}$ Chloralamid. Pat. tobt ununterbrochen weiter, erhält um $12 \mathrm{Uhr}$ wieder $2 \mathrm{~g}$ Chloralamid, wird etwas ruhiger schläft von 2-6 Uhr Morgens mit geringen Unterbrechungen. 8 Uhr Temp. 36,8, Puls 96, Resp. 20. 9 Uhr Temp. 37,0, Puls 106, Resp. 18 . 1 Uhr Temp. 37,0, Puls 102, Resp. 18.

XVII. Kallewe, 34jähriger Mann. Delirium tremens.

25. September. Vormittags $11 \mathrm{Uhr} 2 \mathrm{~g}$ Somnal. Keine Wirkung. Mittags $1 \mathrm{Uhr} 2 \mathrm{~g}$ Somnal, Pat. wird etwas ruhiger, aber schläft noch gar nicht. Abends 6 Uhr $2 \mathrm{~g}$ Somnal. Pat. bricht fortwährend, wird aber ruhig. Er schläft nur von Morgens $4-6$ Uhr. Puls un 11 Uhr Vormittags 96, um 10 Uhr Abends 102.

26. September. Pat. schläft Vormittags fortwährend, klagt über Uebelkeit und Kopfschmerzen.

27. September. Da Pat. wieder unruhig wird, aus dem Bette klettert etc., werden ihm um 8 Uhr $2 \mathrm{~g}$ Chloralamid verabfolgt. Pat. schläf um $9 \mathrm{Uhr}$ ein und wacht erst Morgens $6 \mathrm{Uhr}$ wieder auf.

\section{Gruppe: Nervöse Agrypnie i. e. S. (ohnebesondere Auf- regungszustände).}

XVIII. Holz, 63 jähriger Mann. Dementia. Schlaflosigkeit.

2. September. Abends 8 Uhr 2 g Somnal. Pat. schläft. $8 \%$ Uhr ein schläft mit Unterbrechungen bis Morgens $6 \mathrm{Uhr}$, Schlaf sehr leise. 8 Uh Temp. 37,3, Puls 70, Resp. 16. 9 Uhr Temp. 37,0, Puls 64, Resp. 16 10 Uhr Temp. 36,6, Puls 64, Resp. 16. Urin spec. Gewicht 8 Uhr Abends

1012. Urin spec. Gewicht 8 Uhr Morgens am 7. September 1020.

7. September. Taumelig, K opf schmerzen.

XIX. Merlitz, 23jähriger Mann. Lues secundaria.

23. September. Abends 8 Uhr $2 \mathrm{~g}$ Somnal. Pat. schläft die ganze Nacht fast ohne Unterbrechung. Schlaf leise.

24. September. Vormittags Erbrechen und Kopfschmerzen.

26. September. Abends 8 Uhr $2 \mathrm{~g}$ Chloralamid. Pat. wacht öfters auf, hat fortwährend Angstgefühl.

28. September. Schläft den ganzen Vormittag. Nachmittags Gang schwankend, behauptet, dass die gange Stube in Kreise herumgehe.

XX. Treue, 19 jähriger Wann. Gut compensirter Herzfehler.

8. September. Ábends 8 Uhr $2 \mathrm{~g}$ Somnal. Schläft gut.

9. September. Hat etwas Kopfschmerzen. Abends 8 Uhr $2 \mathrm{~g}$ Somnal. Schläft wieder ohne Unterbrechung bis Morgens 6 Uhr.

10. September. Fühlt sich müde. Abends 8 Uhr $2 \mathrm{~g}$ Somnal. Schläft ununterbrochen die ganze Nacht.

11. September. Hat Magenschmerzen, kann nichts geniessen.

15. September. Abends $8 \mathrm{Uhr} 2 \mathrm{~g}$ Chloralamid. Pat. schläft $8^{1 / 2} \mathrm{Uhr}$ rut und erwacht erst Morgens $9 \mathrm{Uhr}$

16. September. Starke Kopfschmerzen und Magenschmerzen, weigert sich, dis Mittel noch einmal zu nehmen.

XXI. Berge:", 60jähriger Mann. Strictura Oesophagi. Keine Schmerzen.

6. September. Abends 8 Uhr $2 \mathrm{~g}$ Somnal. Pat. schläft um $8 \frac{1}{2}$ Uhr ein, wacht um $11^{1 / 2}$ Uhr auf und bleibt bis zum Morgen wach.

7. September. $2 \mathrm{~g}$ Somnal mit gleichein Erfolge.

8. September. Klagt über Müdigkeit und Kopfschmerzen.

10. September. Abends 8 Uhr $2 \mathrm{~g}$ Chloralamid. Pat. schläft gegen 9 Uhr ein, wacht bis 2 Uhr häufig auf: weiterhin ununterbrochener Schla bis zum Morgen.

11. September. Abends $8 \mathrm{Uhr} 2 \mathrm{~g}$ Chloralamid. Schläft bis $2 \mathrm{Uhr}$ ohne Unterbrechung, von da ab gar nicht mehr.

12. September. Heftige Klagen über Magenbesehwerden.

15. September. Abends 8 Uhr $2 \mathrm{~g}$ Chloralhydrat. Bis 2 Uhr fester Schlaf, wacht bis $3^{1} / 2$ Uhr, schläft dann bis 6 Uhr. Fühlt sich am Vormittag des 16. September müde, taumelig.

XXII. Kieser, 34jähriger Mann. Abgelaufene Pleuritis exsudativa.

13. September. Abends $8 \mathrm{Uhr} 2 \mathrm{~g}$ Somnal. Schläft $8^{1 / 2} \mathrm{Uhr}$ ein wacht um 9 Uhr beim Messen auf und kann nicht wieder Schlaf finden. Pat. bekommt um $11 \mathrm{Uhr}$ ein drittes Gramm Somnal, welches ohne jede Wirkung. Morgens 4-6 Uhr schläft Pat. 8 Uhr Temp. 36,8, Puls 84, Resp. 18. 9 Uhr Temp. 36,6, Puls 80, Resp. 18. 10 Uhr Temp. 36,5, Puls 78, Resp. 16. 12 Uhr Temp. 36,5, Puls 96, Resp. 20.

14. September. Vormittags beftige „Kopfstiche“. Abends 8 Uhr $2 \mathrm{~g}$ Somnal. Schläft im ganzen 2 Stunden gegen Morgen.

17. September. Abends 8 Uhr $2 \mathrm{~g}$ Chloralamid. Schläft um $9 \mathrm{Uhr}$ ein, wacht um 2 Uhr erst wieder auf, schlät von $21 / 2$ bis 6 Uhr. Schlaf leise.
18. September. Abends 8 Uhr $2 \mathrm{~g}$ Chloralamid. Schläft leise, weckt beim Messen auf, schläft aber stets oleich wieder ein bis um 6 Uhr Morrens.

19. September. Starke Magenschmerzen. Pat. schläft die Nacht circa 3 Stunden in Unterbrechung.

20. September. Abends 8 Uhr $2 \mathrm{~g}$ Chloralhydrat. Pat. schläft mit einer Unterbrechung die ganze Nacht, weckt beim Messen nicht auf.

XXIII. Albrecht, 47 jähriger Mann. Polyarthritis rheum. chron. ohne Schmerzen.

22. September. Abends 8 Uhr 2 g Somnal. Schläft $8^{1 / 4}$ Uhr ein, wacht beim Messen um 9 Uhr auf, schläft nicht wieder ein. Um $11 \mathrm{Uhr} 1 \mathrm{~g}$ Somnal. Schläft von $11^{1 / 2}$ bis 2 Uhr, dann wach bis zum Morgen.

23. September. Vormittags. „Lieber will ich nicht schlafen, als das Kopfreissen noch einmal durchmachen".

25. September. Abends 8 Uhr $2 \mathrm{~g}$ Chloralamid. Pat. schläft fast ohne Unterbreclıung die ganze Nacht.

26. September. Legt sich um 8 Uhr wegen allzu grosser Müdigkeit hin und muss um 1 Uhr zum Mittagessen geweckt werden. Nachts kann Pat. fast gar nicht schlafen.

27. September. 8 Uhr $2 \mathrm{~g}$ Chloralamid. Schläft wieder die Nacht fast ohne Unterbrechung durch.

28. September. Sehr müde. Klagt über Uebelkeit.

Es liegt mir fern, den Leser mit weiteren Protokollen, die zudem nichts neues ergeben und aus wahen Gründen ebenso wenig des Lückenhaften entbehren würden, zu ermüden und berichte mit Folgendem summarisch:

Ich habe je 120 Einzelversuche bei je 50 Patienten sowohl vom Somnal, als vom Chloralamid angestellt, und zwar bei je 36 Männern und je 14 Frauen. Bei Kindern wurde, da schon bei Erwachsenen die Bedenken, wie aus obigen Aufzeichnungen erhellt, nicht unerhebliche waren, von einem Versuche Abstand genommen.

Von den 50 Patienten, an denen Versuche angestellt wurden, litten 20 an Agrypnie, bedingt durch organische Erkrankung, 6 an acuten Infectionskrankheiten, 6 an Delirium potatorum, 18 an nervöser Agrypnie.

Die speciellen Krankheitsformen anlangend, bestand bei:

8 Delirium tremens,

8 Herzentartung und Klappenfehler,

6 Typhus abdominalis,

6 Phthisis pulmonum,

5 Gelenkrheumatismus (acut und chronisch),

3 Magenkrebs,

3 Nierenentzündungen (acut und chronisch),

3 Pleuritis exsudativa,

2 Magengeschwür,

2 Strictura Oesophagi,

2 Lues secundaria,

1 Dementia

1 Intoxicatio saturnina.

50

Vier alte, vom Marasmus senilis abgesehen, vollkommen gesunde Männer, welche an zwei verschiedenen Tagen Morgens 7 Uhr 2 g Somnal und $2 \mathrm{~g}$ Chloralamid erhielten, verspürten keinerlei Wirkung. Der eine beklagte sich nur, die Nacht darauf (nach Chloralamid) schlechter geschlafen zu haben, wie sonst, da el von beängstigenden Träumen sehr geplagt wurde. Letzterer erhielt einige Tage darauf Morgens 9 Uhr 2 g Chloralhydrat. Er wurde nach dem Mittagessen sehr müde, schlief um 2 Uhr ein und musste Abends 7 Uhr zur Einnahme der Abendmahlzeit geweckt werden.

Bei den 20 Patienten, welche an Agrypnie infolge körperlicher Beschwerden gelitten, hat Somnal 3 mal gut, 5 mal mittelmässig, 1 12 mal gar nicht schlafbringend gewirkt; Chloralamid 2 mal gut, 4 mal mittelmässig, 14 mal gar nicht schlafbringend gewirkt.

Bei den 6 Patienten mit acuten Infectionskrankheiten hat Somnal 2 mal gut, 2 mal mittelmässig, 2 mal gar nicht schlafbringend gewirkt; Choralamid 0 mal gut, 2 mal mittelmässig, 2 mal gar nicht schlafbringend gewirkt.

Bei den 6 Patienten mit Aufregungszuständen hat Somnal 0 mal gut, 2 mal mittelmässig, 4 mal gar nicht schlafbringend gewirkt; Chloralamid 5 mal gut, 2 mal mittelmässig, 1 mal gar nicht schlafbringend gewirkt.

Bei den 18 Patienten, die an nervöser Agrypnie litten, hat Somnal $10 \mathrm{mal}$ gut, $4 \mathrm{mal}$ mittelmässig, $4 \mathrm{mal}$ gar nicht schlafbringend gewirkt; Chloralamid 6 mal gut, 6 mal mittelmässig, 6 mal gar nicht schlafbringend gewirkt

Es hat:

Gesammtresultat also:

Somnal $15 \mathrm{mal}$ gut, $13 \mathrm{mal}$ mittelmässig, $22 \mathrm{mal}$ gar nicht, Chloralamid $13 \mathrm{mal}$ gut, $14 \mathrm{mal}$ mittelmässig, $23 \mathrm{mal}$ gar nicht s'chlafbringend gewirkt.

Beziehungsweise hat Somnal in $44 \%$, Chloralamid in $46 \%$ versagt.

1) Als „mittelmässig“ bezeichne ich einen mehrfach unterbrochenen bezw. nur bis zu etwa einer Stunde dauernden Schlaf. 
Vergleichen wir zunächst die Wirkung der Mittel in Bezug auf den Charakter der Agrypnie, so fällt auf, dass, wenn dieselbe durch Schmerzen bedingt ist, der Procentsatz der güustigen Fälle viel geringer ist (Somnal 15\%, Chloralamid 10\%), als wenn die nicht durch ein Grundleiden bedingte Schlaflosigkeit die Ursache bil$\operatorname{det}$ (Somnal 55,6\%, Chloralamid 33,3\%).

Wir sind deshalb wohl berechtigt, auch hier, wie beim Amylenhydrat, Sulfonal etc., den Schluss zu ziehen, dass die Mittel im wesentlichen nur Hypnotica, nicht aber Narcotica sind. Hiermit im gewissen Einklang steht die oben constatirte Wirkungslosigkeit beider Schlafmittel am Tage.

Von den in der Einleitung genannten zwei Postulaten eines guten Schlafmittels, Sicherheit bezw. Promptheit der Wirkung und Abgang unangenehmer Nebenerscheinungen, wird vom Chloralamid keins erfüllt, vom Somnal kaum ein einziges.

Es ist bis jetzt, soweit ich die Litteratur verfolgen konnte, das Chloralamid als harmlos in Bezug auf die Circulation geschildert worden. Der Blutdruck soll auf normaler Höhe bleiben, die Herzthätigkeit sich nicht wesentlich ändern.

Ich bedaure, hiermit constatiren zu müssen, dass die Versuche im Krankenhause Friedrichshain ganz andere Resultate, nämlich bedenkliche Nebenwirkungen des Chloralamids auf das Herz, bezw. die Circulation ergeben haben. ${ }^{1}$ )

Dreimal musste ich bei uncompensirten Herzfehlern, ganz abgesehen von den Fällen bei acuten Infectionskrankheiten, eine Verschlechterung der Spannung und Frequenz des Pulses (einmal von 62 auf 108 Schläge in der Minute) constatiren.

In zwei Fällen wurde längere Zeit Kampher nöthig, um den bedrohlich kleinen und schnellen Puls wieder zu heben. Gerade bei diesen Patienten habe ich Chloralhydrat auch zur Anwendung gebracht und gefunden, dass der Puls auch etwas kleiner und schneller wurde, dass aber nie der Grad, wie bei ersterem, erreicht wurde.

Ich versuchte nach diesen Misserfolgen statt $2 \mathrm{~g}$ nur die Hälfte zu geben. Eine unangenehme Wirkung auf's Herz wurde hierbei nicht gefunden, aber die Patienten fühlten auch keine Spur von Müdigkeit. Mehr als $2 \mathrm{~g}$ zu verabreichen durfte nicht gewagt werden.

Bei dem Somnal sind in dieser Beziehung die Versuche viel günstiger ausgefallen. Ich habe bei denselben Patienten, bei denen Chloralamid einen schlechten Puls hervorrief, nur eine geringe Verlangsamung des Pulses bemerkt. Derselbe blieb voll und gut, die Kranken schliefen mit Unterbrechungen und fühlten keine Beschwerden. Dies die Wirkung bei $2 \mathrm{~g}$. Auch $3 \mathrm{~g}$ wagte ich in refracta dosi zu geben, fand aber hierbei eine Verschlechterung des Resultates. Der Schlaf war viel leiser und von grösseren Pausen unterbrochen, der Nebenwirkungen auf den Digestionstractus gar nicht zu gedenken.

Das billige Verlangen nach festem Schlaf wurde durch beide Mittel gleich mangelhaft erfüllt. Selbst bei der grössten Vorsicht war es mir öfters unmöglich, wenn ich behufs Controlle des Pulses an das Bett trat, das Aufwachen zu verhindern. Meist schliefen zwar die Patienten gleich wieder ein, aber wiederholt war mit dem Erwachen jeder Schlaf gebannt, bekanntlich ein beim Morph. mur. und Cloralhydrat seltenes Ereigniss.

Auch den Mangel einer unangenehmen Wirkung auf den Digestionstractus muss ich entschieden in Abrede stellen. Die Fälle ausgenommen, wo ein Magenübel als Grundleiden bestand, habe ich bei Somnal 14, bei Chloralamid 9 mal Klagen hierüber gehört. Das Somnal wurde bei einer Dosis von $2 \mathrm{~g}$ nur einmal erbrochen, bei $3 \mathrm{~g} 4 \mathrm{mal}$. Die Kranken fühlten ungefähr $1 / 2$ bis 1 Stunde nach dem Einnehmen Unbehagen und erbrachen dann so plötzlich, dass es ihnen nicht mehr möglich war, nach einem neben ihnen stehenden Gefässe $\mathrm{zu}$ fassen. 2 mal erfolgte das Erbrechen mehrmals so heftig, dass zuletzt nur Schleim und Galle entleert wurden. In den übrigen Fällen kam es nicht zum Erbrechen, es bestanden aber den ganzen nächsten Tag U e belkeit und Magenschmerzen.

Namentlich bei wiederholten Gaben mehrere Tage hinter einander stellte sich A ppetitlosigkeit und fortwährendes Unbehagen im Leibe ein.

Das Chloralamid bewirkte in $2 \mathrm{~g}$-Dosen nicht einmal Erbrechen. 4 Patienten klagten am nächsten Tage über Magenbeschwerden. Bei grösseren Dosen erfolgte 2 mal Erbrechen, doch ist hier die Wirkung wahrscheinlich nicht allein dem Mittel zuzuschreiben, da es sich um 2 Potatoren handelte. Auch bei mehrtägigem Einnehmen habe ich verhältnissmässig selten Klagen über diese Beschwerden gehört.

1) Soeben entdecke ich eine werthrolle Bestätigung dieser aus der klinischen Beobachtung hergeleiteten Ansicht durch das Thierexperiment. Es ist Langgaard (Therapeut. Monatshefte 1889, Heft 10) gewesen, welcher eine deutliche Abnahme der Gefässspannung bei selbst schwachen Graden der Wirkung constatirte und entsprechende Warnungen vor unliebsamen Erfahrungen ausspricht.
Andere Nebenerscheinungen, die wohl bei der grossen Hälfte bei beiden Medicamenten am nächsten Vormittag oft mehr, oft weniger hervortraten, waren $\mathrm{Sch}$ windel, Mattigkeit, Kopfschmerzen. Ja, es wurden diese Beschwerden von mehreren Patienten derart empfunden, dass dieselben lieber sofort entlassen werdeu wollten, als noch einmal "mit dem Zeng geplagt zu werden."

Noch ein recht wichtiger Punkt ist als nicht seltene Nebenwirkung des Chloralamids hervorzuheben, nämlich die bisweilen bedrohlichen und die Nachtruhe der anderen Kranken in geradezu beängstigender Weise störenden Aufregungszustände. Ich habe unter den vorher protocollirten Versuchen einige angeführt. (Fall No. III, XI, XII, XIII.) Im ganzen sind $16 \mathrm{mal}$ Aufregungszustände, 9 bei Männern, 7 bei Weibern, beobachtet worden.

Auch Herr Prof. Fürbringer hat bei der Anwendung des Mittels in seiner consultativen Praxis derartige Wirkung gleichfalls in wenig willkommener Weise angedeutet bis ausgeprägt gefunden.

Die Zustände verlaufen meist so, dass 2-3 Stunden nach der Einnahme die Patienten aufzustehen versuchen und nach den Beobaclıtungen von Prof. Fürbringer, ähnlich wie bei Vergiftungen mit Cocawein, fortwähreud sinnloses Zeug schwatzen, in schweren Fällen Bettflucht und Ambuliren, ganz ähnlich wie beim Säuferdelirium, eintreten. Gegen Morgen pflegt sich dann Schlaf einzustellen, der manchmal auch den Vormittag des nächsten Tages andauert. Allein auch 3 Fälle beobachtete ich, wo die Aufregung noch den ganzen folgenden Tag anhielt, und erst nach 24 Stunden Schlaf eintrat. Hervorzuheben ist, dass diese unangenehme Nebenwirkung schon bei Verabreichung mittelgrosser Dosen auftreten kann.

Es bleibt mir jetzt noch übrig, die Anwendung beider Mittel bei acuten Infectionskrankheiten und dem Delirium potatorum zu besprechen. Somnal erhielten 6 stark fiebernde Patienten mit Unterleibstyphus, die schon mehrere Nächte vorher nicht geschlafen hatten. Als Ergebniss zeigte sich, dass $1 \mathrm{~g}$ keine Wirkung erzielte, $2 \mathrm{~g}$ in einem Drittel der Fälle mit Erfolg, bei allen ohne jede wesentliche Veränderung der Herzthätigkeit verlief. Dagegen hat die Verabfolgung von $3 \mathrm{~g}$ gerade eine dem Zweck der Ordination entgegengesetzte Wirkung; die Patienten wurden unruhiger, benommener und klagten über grosses Angstgefühl. Am nächsten Tage trat fast stets Erbrechen und starke Eingenommenheit des Kopfes hervor. Der Puls blieb auch bei dieser Dosis voll und kräftig, wurde allerdings gewöhnlich um einige Schläge in der Minute häufiger. Immerhin ist hier $\mathrm{zu}$ betonen, dass die relativ günstige Wirkung des Somnals hinter derjenigen des Sulfonals, wie sie bei anderer Gelegenheit früher im hiesigen Krankenhause constatiert worden ist ( $\mathrm{Sch}$ walbe, Deut. med. Wochenschrift 1888), zurücktritt.

Das Chloralamid wurde wegen der Gefahr eintretender Herzschwäche nur mit aller Vorsicht vier kräftigen Typhuskranken zu reichen gewagt, mit $1 \mathrm{~g}$ begonnen, ohne eine besondere Wirkung erkennen zu lassen; höchstens 2 Stunden fanden die Patienten leichten Schlaf. Eine Einwirkung auf das Herz war nicht zu erkennen. Ich stieg dann auf $1^{1 / 2} \mathrm{~g}$ und konnte regelmässig schon bei dieser Dosis Aufreguugszustände constatieren. Der Puls war etwas beschleunigt, aber voll. Trotzdem versuchte ich in refracta dosi $2 \mathrm{~g}$ Chloralamid zu geben. Die Erfolge waren recht schlechte. Der Puls stieg wieder bedenklich und wurde in einem Falle so schlecht, dass Kampher zur Anwendung kommen musste. Die Patienten wurden sehr unruhig und konnten nur mit Mühe im Bette gehalten werden.

Bei Aufreguugszuständen wurde Somnal bis zu 6 g innerhalb 12 Stunden gegeben. Der Erfolg war aber selbst nach dieser Dosis so gering, dass ein günstiger Einfluss dieses Mittels bei solchen Krankheiten verneint werden muss. Ich muss indess noch einmal hervorheben, dass selbst bei einer Tagesdose von $6 \mathrm{~g}$ kein wesentlicher Einfluss auf den Puls bemerkt wurde.

Das Chloralamid gab ich bis zu $4 \mathrm{~g}$ (in zweistündlichen Gaben von je 2 g) und konnte unter 8 Fällen, die ich beobachtete, 5 gute Erfolge verzeichnen. Bei diesen Zuständen scheint das Chloralamid ebenso promptzu wirken, wie das Chloralhydrat. Auch konnte ich hier nicht ein einziges Mal eine üble Einwirkung auf den Puls feststellen.

Fassen wir nun noch einmal kur\% die praktisch werthvollen Resultate unserer Versuche zusammen, so glauben wir zu folgender Rubricirung berechtigt zu sein: Somnal:

Schlafwirkung:

Unsicher, durchschnittlich als annehmbare nur beobachtet in: $30 \%$

Nebenwirkungen:

Auf die Herzthätigkeit:

Fast fehlend.

Uuter Umständen sehr bedenklich.

Auf den Digestionsapparat: \} relativ häufig in unangenehmer Weise Auf das Allgemeinbefinden:

Geschmack: eben erträglich. hervortretend.

Preis: sehr niedrig. 
So hat denn die - einzig maassgebende - klinische Prüfung, bei aller Anerkennung gewisser schätzenswerther und rationeller Eigenschaften des Somnals und Chloralamids, ein Ueberwiegen der Vortheile leider nicht ergeben.

Wenn auch ohne weiteres zugegeben werden muss, dass eine grössere und noch buntere Auswahl der Versuchspatienten (Irre konnten keine Berücksichtigung finden) das Ergebniss nach dem Günstigeren hin versclioben haben könnten - die Angaben voll Jastrowitz, P. Guttmann u. a. deuten darauf -, so dürfte doch der hohe Procentsatz von Versagern und unliebsamen bis bedenklichen Nebenwirkungen eine Substitution der altbewährten Hypnotica durch die beiden neuen. Schlafmittel ${ }^{\text {" }}$ nicht zulassen. In dieser Erwägung hat Herr Prof. Für rbringer auch letztere als ständige Heilmittel von seiner Abtheilung verbannt, ihre Anwendung für bestimmte durch vorstehende Erfahrungen charakterisierte Einzelfälle reservirend. 